\title{
A notch stress method for fatigue life prediction of spot-welded joints
}

\author{
Changjian $\mathrm{Wei}^{1}$ and Hong Tae Kang ${ }^{1}$ \\ ${ }^{1}$ The University of Michigan, Mechanical Engineering Department, 4901 Evergreen Road, Dearborn, \\ MI, USA 48128
}

\begin{abstract}
Spot-welded joints are widely used in the construction of vehicle structures and frequently become critical locations for fatigue failure. Hence, it is essential to have reliable fatigue life prediction method for the spotwelded joints during vehicle structure design. In this paper, a new notch stress approach is developed for fatigue life prediction of the spot-welded joints. Currently, structural stress methods are widely used in automotive industry for fatigue life prediction of spot-welded joints. However, these methods are not well considering local geometry information. This paper introduces a notch stress based method to overcome the limitation of the structural stress methods. In the notch stress method, stress concentration factors for spot-welded joints are calculated from stress intensity factor equations. Then, the notch stress method is validated with fatigue test results of lap-shear and coach peel specimens.
\end{abstract}

\section{Introduction}

Spot-welded joints in thin-sheet materials are a common joining method in the structural components of automobiles: car body, chassis components, truck cab, and van box. A conventional car body contains several thousands of spot welds. Since about $90 \%$ of cracks occurrent in car bodies in service are originated from spot-welded joints [1], the evaluation methods of fatigue failure of spot-welded joints are very important during vehicle structure design processes. Many researchers [2-19] had proposed the fatigue life prediction methods for spot-welded joints.

It is a very well-known factor that stress values in finite element analysis (FEA) depend on the mesh sizes. Thus, it is required fine-meshed spot-welded joints in the FEA model of a vehicle structure to obtain accurate stress conditions at spot-welded joints. However, it is almost impossible to represent the spot-welded joints of a vehicle structure in fine-meshed FEA models since a vehicle structure contains more than 3,000 spot-welded joints [21]. Thus, structural stress approaches [2-12] were introduced to predict fatigue life of spot-welded joints. These methods calculate the stress at/around the spot-welded joints from the forces and moments obtained from coarse-meshed FEA. Structural stresses are, then, represented as the fatigue damage parameter in stress-life $(\mathrm{S}-\mathrm{N})$ curves.

\footnotetext{
1 Corresponding author: htkang@ umich.edu
} 
Even though the structural stress approaches contribute to eliminate the mesh dependency issue of spot-welded joints, these methods still contain limitations. The structural stress approaches ignore the local geometric effect on fatigue life of spot-welded joints. The structural stresses are defined as being linearized over the plate thickness, which can be considered as a nominal stress. Thus, the local effect due to the notch around the spot-welded joint is completely neglected. Therefore, these methods need extra empirical parameters for better correlation with test results.

This study is aiming to resolve this issue in the structural stress approach by using notch stress concepts. The structural stress equations introduced by Rupp and co-workers [2] are used for the main equations but stress concentration factors (SCF) for each loading mode are multiplied to the structural stress terms. It is assumed that (a) the SCFs are strongly related with the geometric dimensions of the spot-welded joints and the loading modes; (b) the SCFs can be calculated based on a coarse-meshed model in FEA. The notch stress approach developed in this study is compared with the structural stress approach of Rupp and coworkers [2] using the test results of lap-shear specimens and coach peel specimens [20]. The notch stress approach showed better correlation without any adjustment of empirical parameters.

\section{Structural stress equations}

The structural stress in Rupp and co-workes [2] is contributed from three parts: membrane stress due to in-plane shear forces, bending stress due to bending moments and bending stress due to opening forces acting perpendicular to the spot weld plane as shown in Fig. 1. The structural stresses along the circumference of the weld are calculated as below.

$$
\sigma_{\text {stru }}=-\sigma_{\max }\left(F_{X}\right) \cos \theta-\sigma_{\max }\left(F_{Y}\right) \sin \theta+\sigma\left(F_{Z}\right)+\sigma_{\max }\left(M_{X}\right) \sin \theta-\sigma_{\max }\left(M_{Y}\right) \cos \theta
$$

where

$$
\begin{gathered}
\sigma_{\max }\left(F_{X}\right)=\frac{F_{X}}{\pi d t} \times S F F X Y \times d^{D E F X Y} \times t^{T E F X Y} \\
\sigma_{\max }\left(F_{Y}\right)=\frac{F_{Y}}{\pi d t} \times S F F X Y \times d^{D E F X Y} \times t^{T E F X Y} \\
\sigma\left(F_{Z}\right)=\frac{1.744 F_{Z}}{t^{2}} \times S F F Z \times d^{D E F Z} \times t^{T E F Z} \text { if } F_{Z}>0 \text { or } 0 \text { if } F_{Z} \leq 0 \\
\sigma_{\max }\left(M_{X}\right)=\frac{1.872 M_{X}}{d t^{2}} \times S F M X Y \times d^{D E M X Y} \times t^{T E M X Y} \\
\sigma_{\text {max }}\left(M_{Y}\right)=\frac{1.872 M_{Y}}{d t^{2}} \times S F M X Y \times d^{D E M X Y} \times t^{T E M X Y}
\end{gathered}
$$

$t$ is the sheet thickness and $d$ is the diameter of the weld nugget. Equations (2)-(6) are modified from Rupp and co-workers [2] by Fermer and Svensson [21]. SFFXY, SFFZ and $S F M X Y$ are scale factors used to adjust the contributions of the shear forces, axial forces and bending moments due to different loading geometries. DEFXY, DEFZ, DEMXY, TEFXY, $T E F Z$, and $T E M X Y$ are the factors used to adjust the size effect in terms of nugget diameter and sheet thickness. These nine parameters are determined by experimental data. 


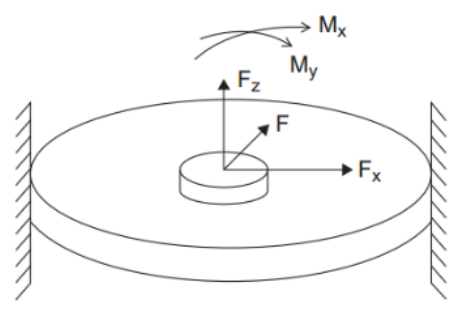

Fig. 1. Circular plate model for a spot-welded specimen [2].

\section{Notch stress calculation}

The goal of this work is to replace the nine emperical parameters in the equations (2)-(6) to stress concentration factors (SCF). However, it is not easy to obtain the SCFs of the spotwelded joints. It is known that the notch stress is directly related to the stress intensity factors (SIF) around the crack tip. Therefore, the first step of obtaining the SCFs is to calculate the SIFs of the spot-welded joint.

\subsection{SIFs solutions for tensile-shear specimen}

According to the works of Radaj [13] and Radaj and Zhang [15], loads of various types of specimens containing spot welds can be decomposed into several symmetric and antisymmetric parts based on the superposition principle of the linear elasticity theory. Fig. 2. schematically shows the decomposition of the shear load of the central part of a tensile-shear specimen. In these schematics, two strips of the sheets with the thickness " $t$ " represent the upper and lower sheet and the gray area with a size of $d=2$ a represents the spot weld.

For the anti-symmetrical membrane loading condition, the SIF solution is:

$$
K_{I I}=\frac{F}{2 \pi d \sqrt{t}}
$$

For the anti-symmetrical bending loading condition, the SIF solution is:

$$
K_{I I}=\frac{3}{\pi} \frac{(F t / 2) \sqrt{t}}{d t^{2}}
$$

For symmetrical bending loading condition, the SIF solution is:

$$
K_{I}=\frac{2 \sqrt{3}}{\pi} \frac{(F t / 4) \sqrt{t}}{d t^{2}}
$$

\subsection{SIFs solutions for coach peel specimen}

Fig. 3. schematically shows the decomposition of the peel loading of a coach peel specimen. For symmetrical bending loading condition, the resultant moment acting on the spot weld nugget is $\mathrm{M}$, and the SIF solution is:

$$
K_{I}=\frac{2 \sqrt{3} M}{\pi d t \sqrt{t}}
$$

Note that the opening force causes a counter bending moment that contributes the SIF solution as:

$$
K_{I}=\frac{\sqrt{3}}{\pi} \frac{F_{z} \sqrt{t}}{t^{2}}
$$



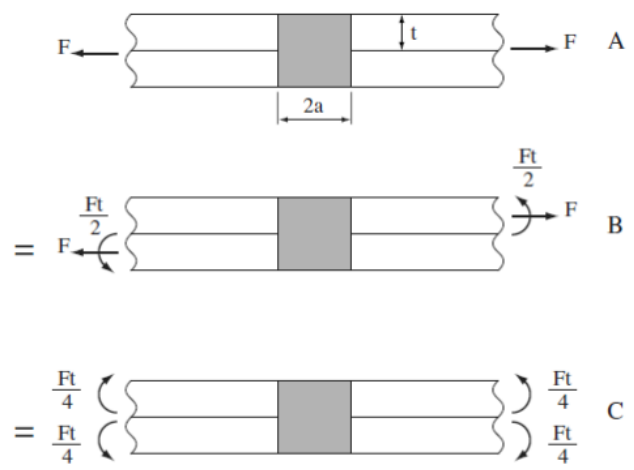

$+$

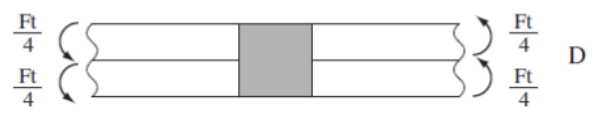

$+$
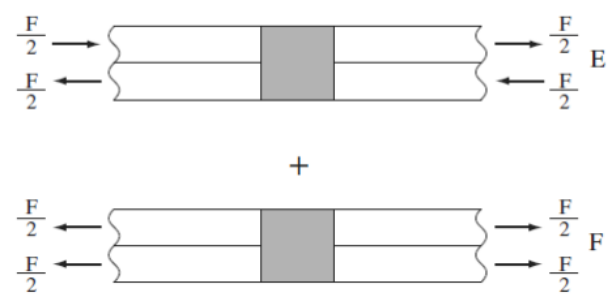

Fig. 2. Decomposition of tensile-shear specimen.

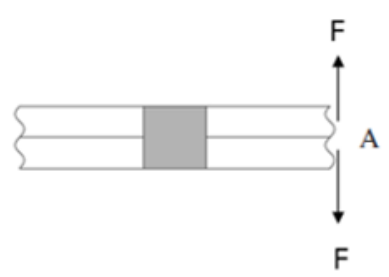

M

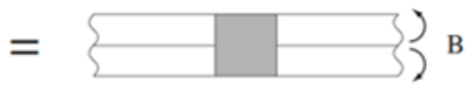

M

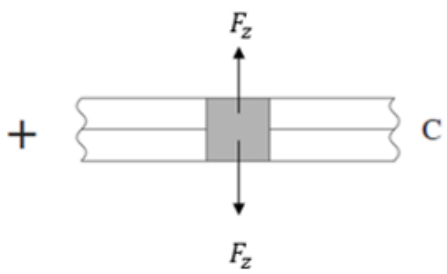

Fig. 3. Decomposition of coach peel specimen.

\subsection{SIFs under mixed mode loading conditions}

The slit tips of a spot weld in a vehicle structure are exposed to mixed mode loading conditions. Thus, an equivalent stress intensity factor $\left(K_{e q}\right)$ concept should be used for the spot-welded joints in a vehicle structure. Yuuki et al. [18] successfully used Erdogan and Sih's equations [16] as shown below for spot-welded joints.

$$
\begin{gathered}
K_{e q}=K_{I} \cos ^{3}\left(\frac{\theta_{\max }}{2}\right)-3 K_{I I} \cos ^{2}\left(\frac{\theta_{\max }}{2}\right) \sin \left(\frac{\theta_{\max }}{2}\right) \\
\theta_{\max }=2 \operatorname{atan}\left(\frac{1}{4}\left(\gamma \pm \sqrt{\gamma^{2}+8}\right)\right), \gamma=\frac{K_{I}}{K_{I I}}
\end{gathered}
$$

If a polar coordinate system is set on the origin of the crack tip, the tangential stress and shear stress are expressed as:

$$
\begin{gathered}
\sigma_{\theta}=\frac{1}{\sqrt{2 \pi r}}\left[K_{I} \cos ^{3} \frac{\theta}{2}-3 K_{I I} \cos ^{2} \frac{\theta}{2} \sin \frac{\theta}{2}\right] \\
\tau_{r \theta}=\frac{1}{\sqrt{2 \pi r}}\left[K_{I} \cos ^{2} \frac{\theta}{2} \sin \frac{\theta}{2}-K_{I I} \cos \frac{\theta}{2}\left(3 \cos ^{2} \frac{\theta}{2}-2\right)\right]
\end{gathered}
$$


When shear stress $\tau_{r \theta}=0$, the tangential stress $\sigma_{\theta}$ reaches the maximum value. From equations (12) and (14), $\sigma_{\theta \max }$ is related with $K_{e q}$. If the notch radius $\rho$ is defined, then the equivalent notch stress $\sigma_{e q}$ is calculated based on the maximum tangential stress as below:

$$
\sigma_{e q}=\frac{K_{e q}}{\sqrt{2 \pi \rho}}=\frac{1}{\sqrt{2 \pi \rho}}\left[K_{I} \cos ^{3}\left(\frac{\theta_{\max }}{2}\right)-3 K_{I I} \cos ^{2}\left(\frac{\theta_{\max }}{2}\right) \sin \left(\frac{\theta_{\max }}{2}\right)\right]
$$

\subsection{Stress concentration factor $K_{t}^{m}$}

Stress concentration factors are defined as the ratio of the equivalent notch stress and the nominal stress:

$$
K_{t}=\frac{\sigma_{e q}}{\sigma_{n}}
$$

In the case of membrane only case, $K_{I I}$ is due to the anti-symmetrical membrane:

$$
K_{I I}=\frac{F}{2 \pi d \sqrt{t}}
$$

Since there is no $K_{I}$, the corresponding equivalent notch stress is:

$$
\sigma_{e q}^{m}=\frac{-3 K_{I I} \cos ^{2}\left(\frac{\theta}{2}\right) \sin \left(\frac{\theta}{2}\right)}{\sqrt{2 \pi \rho}}
$$

Thus, SCF for the membrane only case is defined as:

$$
K_{t}^{m}=\frac{\sigma_{e q}^{m}}{\sigma_{n}^{m}} \approx 0.23 \sqrt{\frac{t}{\rho}}
$$

\subsection{Stress concentration factor $K_{t}^{Z}$}

In the case of bending due to $F_{z}$, SIF $K_{I}$ is induced from the symmetric bending:

$$
K_{I}=\frac{\sqrt{3}}{\pi} \frac{F_{Z} \sqrt{t}}{t^{2}}
$$

Since there is no $K_{I I}$, the corresponding equivalent notch stress from equation (16) is:

$$
\sigma_{e q}^{Z}=\frac{K_{I} \cos ^{3}\left(\frac{\theta}{2}\right)}{\sqrt{2 \pi \rho}}
$$

$\theta$ depends on the ratio of $K_{I}$ and $K_{I I}$, according to Eq. (13). The stress for bending is given by Eq (16), and SCF for bending due to $F_{z}$ is:

$$
K_{t}^{Z}=\frac{\sigma_{e q}^{z}}{\sigma_{n}^{z}} \approx 0.23 \sqrt{\frac{t}{\rho}}
$$

\subsection{Stress concentration factor $K_{t}^{b}$}

In the case of bending due to moment $M$, it should be emphasized that the moment is consisted of symmetrical bending and anti-symmetrical bending. According to the assumption that the general loading condition can be constituted by superposing the loading on the tensile-shear and coach peel. The SIF due to the symmetrical bending is defined as: 


$$
K_{I}^{N}=\frac{2 \sqrt{3}}{\pi} \frac{\left(M-\frac{F t}{2}\right) \sqrt{t}}{d t^{2}}
$$

Note that $\mathrm{M}$ is obtained from FEA as the nodal moment acting on the spot weld. The selfbalanced moment is not included in Eq (24) since FEA cannot capture it. The SIF induced by the self-balanced bending is defined as :

$$
K_{I}^{\text {Self }}=\frac{2 \sqrt{3}}{\pi} \frac{(F t / 4) \sqrt{t}}{d t^{2}}
$$

The SIF due to anti-symmetric bending is also defined as:

$$
K_{I I}^{N}=\frac{3}{\pi} \frac{(F t / 2) \sqrt{t}}{d t^{2}}
$$

Then, combine Eqs (16), (24), (25) and (26), the corresponding equivalent notch stress becomes:

$$
\sigma_{e q}^{b}=\frac{K_{I}^{N} \cos ^{3}\left(\frac{\theta}{2}\right)-3 K_{I I}^{N} \cos ^{2}\left(\frac{\theta}{2}\right) \sin \left(\frac{\theta}{2}\right)}{\sqrt{2 \pi \rho}}+\frac{K_{I}^{\text {Self }} \cos ^{3}\left(\frac{\theta}{2}\right)}{\sqrt{2 \pi \rho}}
$$

Finally, SCF for bending moment $\mathrm{M}$ is:

$$
K_{t}^{b}=\frac{\sigma_{e q}^{b}}{\sigma_{n}^{b}}
$$

Therefore, the notch stress of the spot-welded joint is defined as:

$$
\sigma_{k}=K_{t}^{m} \frac{F}{\pi d t}+K_{t}^{b} \cdot \frac{6 M}{\pi d t^{2}}+K_{t}^{Z} \cdot \frac{3 F_{z}}{\pi t^{2}}
$$

It's worth mentioning that $K_{t}^{m}, K_{t}^{b}, K_{t}^{Z}$ are the functions of $\left(\frac{D}{d}, \sqrt{\frac{t}{\rho}}\right)$. According to Radaj [19], a notch radius of $\rho=0.05 \mathrm{~mm}$ is recommended in low-carbon steels. Boundary condition correction coefficients can be added to these three trems. And the correction coefficients are the function of $\frac{D}{d}$. Here $\mathrm{d}$ is the spot weld nugget diameter and $D$ is the effective diameter of spot-welded joint, which is defined as the square-root value of the overlapped area of the joint.

\section{Application of notch stress approach}

Fatigue data from A/SP [20] for steel to steel single spot-welded joints were used in this study for validation. The specimen configuration is shown in Fig. 4. All the steel to steel fatigue data of load range versus cycles to failure are plotted on the same graph as shown in Fig. 5.

In order to illustrate the improvement of notch stress method, a comparison to the nCode structural stress method are made. For the nCode approach, the default values of nine parameters were used and the result is shown in Fig. 6. From Fig. 6 visible separation is observed between coach peel and lap-shear fatigue data. To collapse two groups of the data, a new set of nine parameters are needed.

The results of notch stress method for the same fatigue test data are plotted in Fig. 7. Two data are collapsing well. 


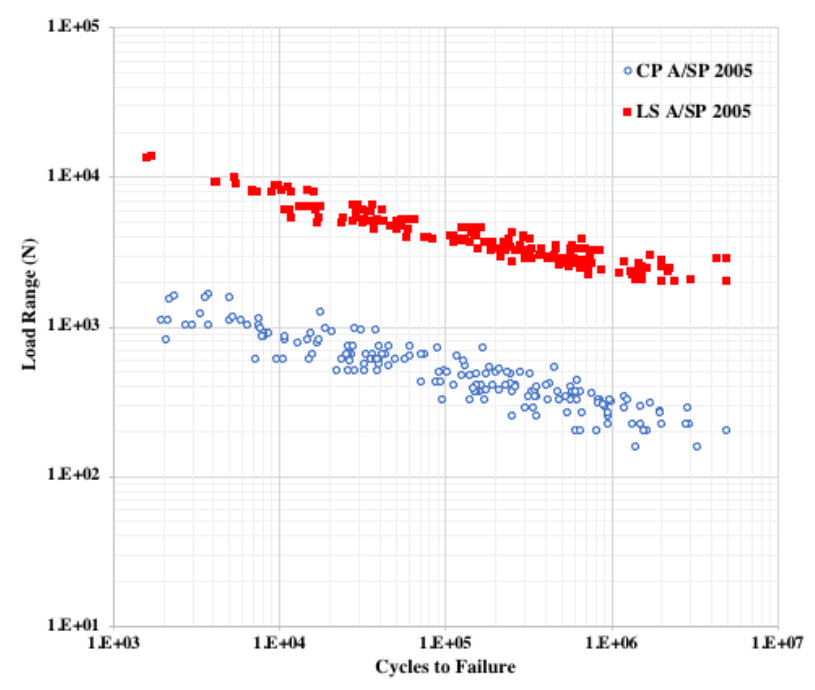

Fig. 4. Load range versus cycles to failure for spot-welded joints.

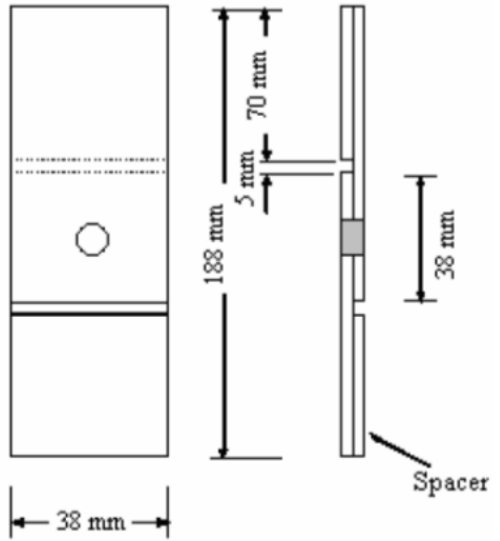

Lap-Shear specimen

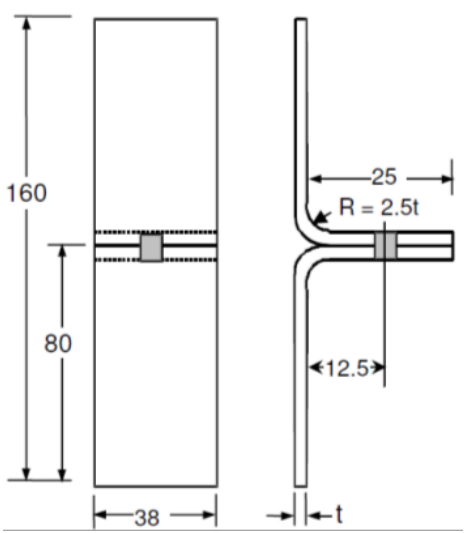

Coach Peel specimen

Fig. 5. Specimen configuration for spot-welded joints.

\section{Conclusions}

The new notch stress approach used three stress concentration factors (SCFs) to eliminate the nine empirical parameters used in nCode structural stress equations. The SCFs are calculated from SIFs in front of crack tip. The self-balanced force and moment were also taken into account in the notch stress approach. Therefore, notch stress approach works better than nCode structural stress method for the fatigue test results of the lap-shear and coach peel specimens.

Authors are very thankful for General Mortors for supporting the project. Especially, Dr. AK Khosrovaneh's valuable discussions are appreciated. 


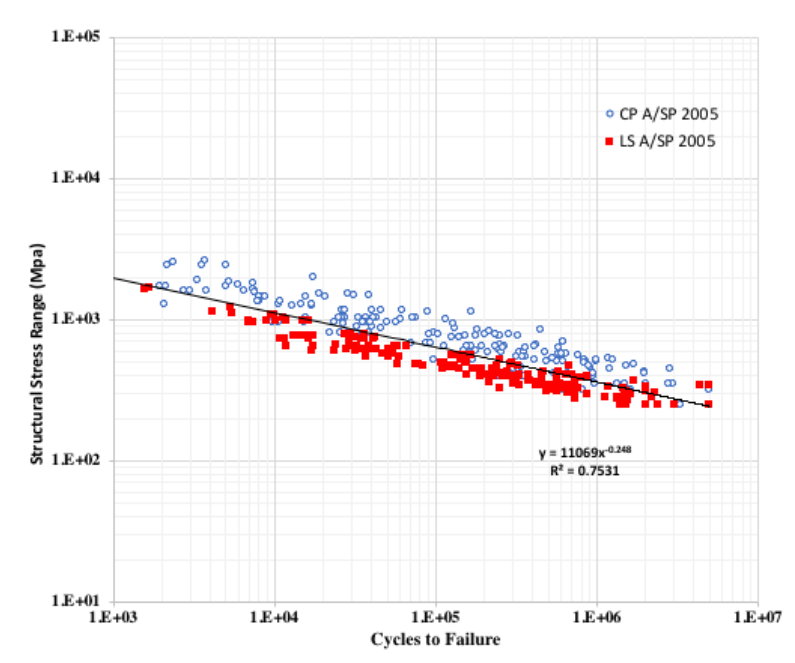

Fig. 6. nCode structural stress range vs cycles to failure.

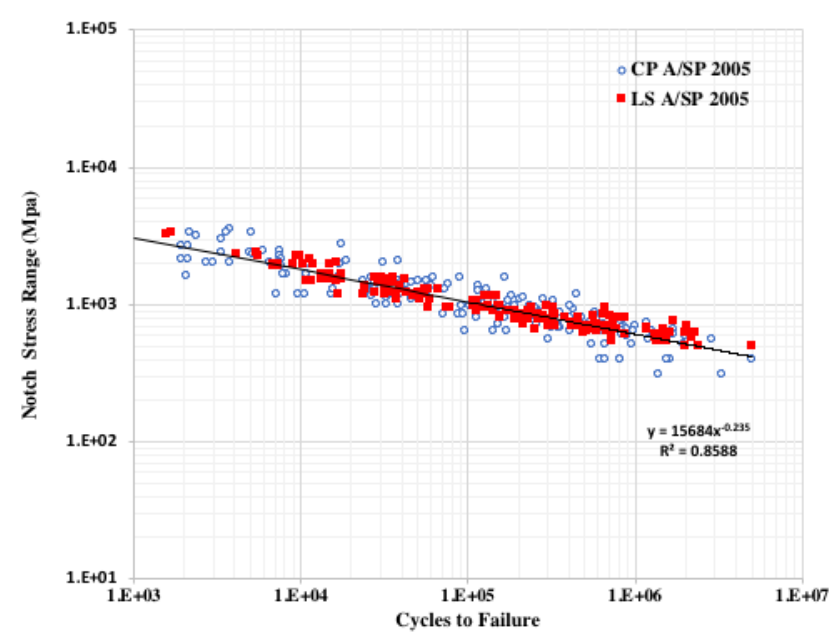

Fig. 7. Notch stress range vs cycles to failure.

\section{References}

1. D. Radaj, C. M. Sonsino, W. Fricke, Fatigue Assessment of Welded Joints by Local Approaches (Abington Publishing, Cambridge, UK, 2006)

2. A. Rupp, K. Störzel, V. Grubisic, 'Computer-aided dimensioning of spotwelded automotive structures,' SAE 950711 (1995)

3. Y. Nakahara, M. Takahashi, A. Kawamoto, M. Fujimoto, et al., 'Method of Fatigue Life Estimation for Spot-Welded Structures,' SAE 2000-01-0779 (2001)

4. S. D. Sheppard, 'Estimation of fatigue propagation life in resistance spot welds', Advances in Fatigue Lifetime Predictive Techniques, ASTM STP 1211, 2 (1993) 
5. S. D. Sheppard, 'Further refinement of a methodology for fatigue life estimation in resistance spot weld connections', Advances in Fatigue Lifetime Predictive Techniques, ASTM STP 1292, 3 (1996)

6. S. D. Sheppard, M. Strange, 'Fatigue life estimation in resistance spot welds: initiation and early growth phase,' Fatigue Fract Engng Mater Struct, 15, 6 (1992)

7. D. Radaj, 'Stress singularity, notch stress and structural stress at spot welded joints,' Engng Fracture Mech. 34 (1989)

8. D. Radaj, 'Theory of forces and stresses in spot-welded overlap joints,' Archive Appl Mech, 67 (1996)

9. P. Dong, J. K. Hong, Z. Cao, A Mesh-Insensitive Structural Stress Procedure for Fatigue Evaluation of Welded Structures, IIW Doc. XIII-1902-01/XV1089-01 (2001)

10. J. K. Hong, 'The development of a simplified spot weld model for Battelle structural stress calculation,' SAE 2011-01-0479 (2011)

11. H. Kang, P. Dong, J. K. Hong, 'Fatigue analysis of spot welds using a meshinsensitive structural stress approach,' Int. J. Fatigue, 29 (2007)

12. S. J. Maddox, 'Fatigue Design of Welded Structures,' Engineering Design in Welded Constructions (Pergamon, Oxford, 1992)

13. S. Zhang, 'Stress intensities derived from stresses around a spot weld,' Int. J. Fract. 99 (1999)

14. D. Radaj, S. Zhang, 'Stress intensity factors for spot welds between plates of unequal thickness,' Eng. Fract. Mech. 39 (1991)

15. S. Zhang, 'Stress intensities at spot welds,' Int. J. Fract. 88 (1997)

16. F. Erdogan, G. C. Sih, 'On the crack extension in plates under plane loading and transverse shear,' J. Basic Engng 85 (1963)

17. R. Yuuki, T. Ohira, H. Nakatsukasa, W. Yi, 'Fracture mechanics analysis and evaluation of the fatigue strength of spot welded joints,' Trans. Japan. Soc. Mech. Engrs. 51-467 (1985)

18. D. Radaj, H. P. Lehrke, S. Greuling, 'Theoretical fatigue-effective notch stresses at spot welds,' Fatigue Fract Engng Mater Struct, 24 (2001)

19. P. -C. Lin, J. Pan, 'Closed-form structural stress and stress intensity factor solutions for spot welds in commonly used specimens,' Engineering Fracture Mechanics, 45 (2008)

20. J. Bonnen, H. Agrawal, M. Amaya, R. Iyengar, et al., 'Fatigue of Advanced High Strength Steel Spot-Welds,' SAE 2006-01-0978 (2006)

21. M. Fermér, H. Svensson, 'Industrial experiences of FE-based fatigue life predictions of welded automotive structures,' Fatigue Fract Engng Mater Struct, 24 (2001) 